\title{
Simultaneous measurements of aeroacoustic sounds and wall vibration for exploring the contribution of tooth vibration in the production of sibilant sounds/s/
}

\author{
Masanori Nakamura ${ }^{1}$, Kazunori Nozaki ${ }^{1}$, Haruka Takimoto ${ }^{2}$, Kazuya Nagamune ${ }^{2}$, \\ Motoharu Fujigaki ${ }^{3}$, Shigeo Wada ${ }^{2}$
}

\footnotetext{
${ }^{1}$ The Center for Advanced Medical Engineering and Informatics, Osaka University, Toyonaka, Japan;

${ }^{2}$ Department of Mechanical Science and Bioengineering, Osaka University, Toyonaka, Japan;

${ }^{3}$ Department of Opto-Mechatronics, Faculty of Systems Engineering, Wakayama University, Wakayama, Japan.

Email:masanori@me.es.osaka-u.ac.jp
}

Received 3 December 2010; revised 23 December 2010; accepted 28 December 2010

\begin{abstract}
In order to understand the contribution of teeth vibration to the production of sibilant $/ \mathrm{s} /$, the present study was designed to develop a method of simultaneously measuring aeroacoustic sounds and the vibration of an obstacle. To measure the vibration without disturbing flow, the Michelson interferometer was employed. The flow channel, which had an obstacle wall inside of it, was fabricated such that it morphologically mimicked the simplified geometry of the oral cavity. Given airflows at a flow rate of $7.5 \times 10^{-4} \mathrm{~m}^{3} / \mathrm{s}$ from the inlet, aeroacoustic sounds were generated. A spectrum analysis of the data demonstrated two prominent peaks in the sound at 1,300 and $3,500 \mathrm{~Hz}$ and one peak in the wall vibration at $3,500 \mathrm{~Hz}$. The correlation in peak frequencies between the sound and wall vibration suggests that the sound at $3,500 \mathrm{~Hz}$ was induced by the wall vibration. In fact, the sound amplitude at $3,500 \mathrm{~Hz}$ decreased when the obstacle wall was thickened, which increased its rigidity $(p<$ $0.05, t$-test). The experimental results demonstrate that the developed techniques are capable of measuring aeroacoustic sound and obstacle wall vibration simultaneously, and suggest the potential to pave the way for detailed analysis of the production of sibilant sounds $/ \mathrm{s} /$.
\end{abstract}

Keywords: Acoustic Measurement; Vibration Measurement; Michelson Interferometer; Aeroacoustics; Sibilant/s/

\section{INTRODUCTION}

Oral therapies for speech disorders are directly linked to quality of life (QOL). In oral therapies, the modification of oral morphological features including changes in the spatial position of the jaw is performed surgically for the purpose of maxillofacial orthodontic therapies [1], prosthetic treatments [2], and the insertion of sports mouth-guards [3]. Alterations in oral morphologies, however, often bring about vocal disorders, in particular, dental fricative sounds that are produced within the oral cavity.

Among the dental fricative voices, sibilant $/ \mathrm{s} /$ has gained relatively much attention from dentists and scientists because sibilant sounds $/ \mathrm{s} /$ are frequently used in daily conversations and most languages [4-6]. The sound source of sibilant /s/ is generally accepted to be the anterior teeth $[7,8]$. From a fluid mechanical point of view, when the sibilant $/ \mathrm{s} /$ is produced, a jet that develops through the constriction made by the tongue and maxilla is speculated to impinge on the anterior teeth, yielding flow turbulence and causing chaotic formation of vortices of many different length scales. The interaction of the vortices leads to rapid variation in pressure on the surface of anterior teeth, thereby inducing pressure fluctuations that cause sibilant $/ \mathrm{s} /$.

Sibilant /s/ has been explored experimentally, theoretically, and numerically. For example, Stevens $[9,10]$ applied aerodynamic flow theory to study acoustic mechanisms of fricative sounds. The study was later followed by the work of Shadle $[7,11,12]$, who modeled the oral cavity as a circular duct with constriction and an obstacle. Shadle's model is quite simple, but the obtained results were important in the research of sibilant $/ \mathrm{s} /$. The study measured velocities and sound pressures that radiated from the model by parametrically varying geometrical features. The authors concluded that acoustically, the significant parameters are the length of the front cavity, the presence of an obstacle, and flow 
rate. Theoretical predictions of the far-field sound of sibilant /s/ were performed by Howe and McGowan [13], who used a singularity analysis. Similarly, but numerically, Nozaki et al. [14] predicted far-field sound propagation by implementing large eddy simulation (LES), along with solving the Lighthill-Curle equation $[15,16]$. Van Hirtum et al. [17] studied turbulence using LES and experiments in the aperture formed by the tooth-shaped structure. Although these studies have provided valuable information for understanding the mechanisms underlying the generation and propagation of sibilant $/ \mathrm{s} /$, the obstacle vibration was out of focus in their study. The possibility exists that the teeth may vibrate and therefore contribute to the production of sibilant $/ \mathrm{s} /$.

When exploring the vibration of an obstacle wall as a sound source, one must have undisturbed flows. For this purpose, an optical technique called the Michelson interferometer is suitable. This method has been classically used in many studies, including optical communications and astrophysics (e.g., [18]). In addition, as recognized by pioneering researchers, this measuring system is sensitive to vibrations and the displacement of a target. Theoretically, a spatial resolution of the Michelson interferometer is smaller than a wavelength of light. In this sense, the Michelson interferometer is suitable for studying vibration-induced sounds that necessitate measuring tiny oscillations of the obstacle wall.

The final goal of our project was to identify the aeroacoustic sources of sibilant sounds /s/. As stated above, one must understand the contributions of tooth vibration to the production of sibilant /s/. As a preliminary study, here we developed a method of simultaneously measuring aeroacoustic sounds and the vibration of an obstacle. Using this method, we measured aeroacoustic sounds and the vibration of an obstacle wall from a flow channel that morphologically simplified the oral cavity when the sibilant sound /s/ was produced.

\section{METHOD}

\subsection{Experimental Setup}

Figure 1 conceptually illustrates the experimental setup, which consists of an air compressor (Yaezaki, YC-4RS), a flow channel with an obstacle wall, a microphone (Earthworks, 30BX), the Michelson interferometer (Chuo Precision Industrial Co, Ltd.), and a PC. In this setup, the air compressor delivers air to the flow channel and generates the aeroacoustic sound. The aeroacoustic sound is measured with a microphone. The Michelson interferometer (elaborated more in later paragraphs) is used to measure the frequency of the vibration of the obstacle wall. All of the experimental devices, except the air compressor and PC, were mounted on a vibration-isolated table (Chuo Precision Industrial Co, Ltd., ORR-1890)

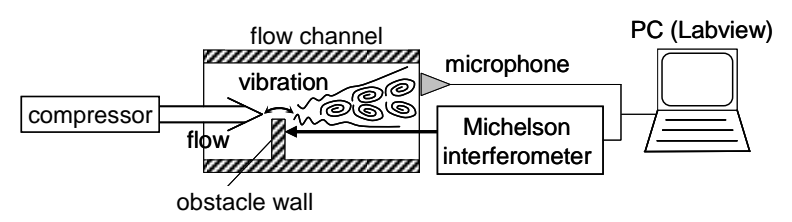

Figure 1. Experimental setup for simultaneous measurements of aeroacoustic sounds and vibration of an obstacle wall.

to minimize the effects of extraneous vibrations on the system.

\subsection{Flow Channel}

The flow channel was based on the design of Shadle [11], who showed that an obstacle in the path of a jet results in localized sound generation. A cross-sectional image of the flow channel is presented in Figure 2. The flow channel mainly consists of a back cavity, a constriction, a front cavity, and an obstacle wall. Morphologically, the flow channel simplifies the geometry of the oral cavity when the sibilant $/ \mathrm{s} /$ is produced, including the back cavity, the constriction and the front cavity representing the pharynges and the oral cavity, the sibilant groove that is produced by the tip of tongue and the upper maxilla, and the space among the lips and teeth, respectively [11]. The cross section of the flow channel perpendicular to the flow direction is circular. The flow channels in the back cavity, the constriction, and the front cavity are connected concentrically to the center of the flow channel. The diameters of the flow channels in the back and front cavities were both $25.4 \mathrm{~mm}$, and the diameter of the constriction was $6.4 \mathrm{~mm}$. The thickness of an obstacle wall was $0.5 \mathrm{~mm}$ in the standard model, but later varied to $0.29,1,2$, and $5 \mathrm{~mm}$ to investigate the influence of thickness. The height of the obstacle wall from the bottom of the flow channels was $12.7 \mathrm{~mm}$, which blocks half of the flow channel. In this situation, the obstacle wall appears to be half-moon-shaped when viewed from the exit of the flow channel. The flow channel was made of chalcopyrite and its internal surface was finely polished to minimize the development of turbulence on the surface such that it satisfies $\mathrm{Ra}=1.6$,

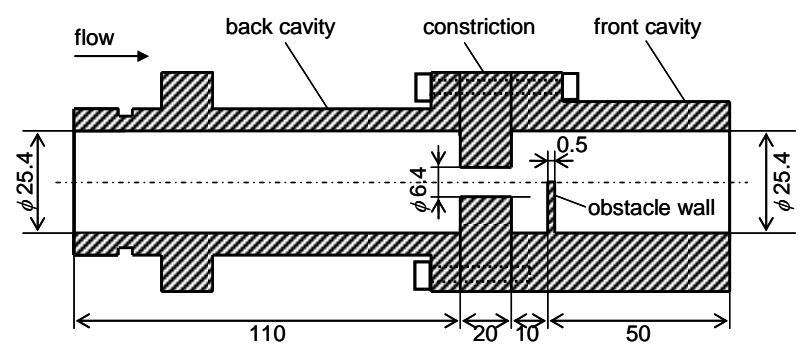

Figure 2. Cross-sectional image of a flow channel. The channel consists of four parts: the back cavity, constriction, obstacle wall, and front cavity. 
$\mathrm{Rz}=6.3$, and $\mathrm{R}_{\mathrm{JIS}}=6.3$ of the Japanese Industrial Standards. Note that this is a scale model, about 3 times as large as an anatomical scale of the oral cavity [19].

\subsection{Wall Vibration Measurement}

Wall vibration frequency was measured using the Michelson interferometer and photomultiplier. Figure 3 shows a schematic drawing of the experimental apparatus for measuring wall vibrations. The experimental apparatus consisted of a He-Ne laser beam oscillator (LO) (Chuo Precision Industrial Co, Ltd., GL5230, true wavelength $632.8 \mathrm{~nm}$, maximum optical output power $20 \mathrm{~mW}$ ), a beam expander unit (BE) (Chuo Precision Industrial Co, Ltd., C-80, $\times 20$ ), a slit unit (SU) (Chuo Precision Industrial Co, Ltd., C-25, $\phi 2-30 \mathrm{~mm}$ ), a beam splitter unit (BS) (Chuo Precision Industrial Co, Ltd., IU-BS1, $\phi 20 \mathrm{~mm}$ ), a mirror unit (MU) (Chuo Precision Industrial Co, Ltd., IU-M1, $\phi 20 \mathrm{~mm}$ ), and a photomultiplier (PM) (Hamamatsu Photonics, light sensor module H9656, effective wavelength $632.8 \mathrm{~nm}$ ). Additionally, a circular mirror with a diameter of $6.3 \mathrm{~mm}$ (Edmund Optics, 4-6 $\lambda$ ) was glued to the upper edge of the obstacle wall for reflecting the laser beam. The experimental parts were precisely positioned as follows: The distance was $330 \mathrm{~mm}$ between the OW and BS, $330 \mathrm{~mm}$ between the MU and BS, $200 \mathrm{~mm}$ between the PM and BS, 220 $\mathrm{mm}$ between the BS and SU, $60 \mathrm{~mm}$ between the SU and $\mathrm{BE}$, and $10 \mathrm{~mm}$ between the SE and LO. The Michelson interferometer produces interference fringes by recombining two beams of light generated from the same beam source. In brief, the beam of light was generated by the He-Ne laser beam oscillator. It was then expanded and made to be parallel by the downstream beam expander and split in two by the semitransparent mirror. From this point, two paths of light went to the detector. One reflected off the semitransparent mirror, struck the bottom mirror, and then bounced back, passing through the

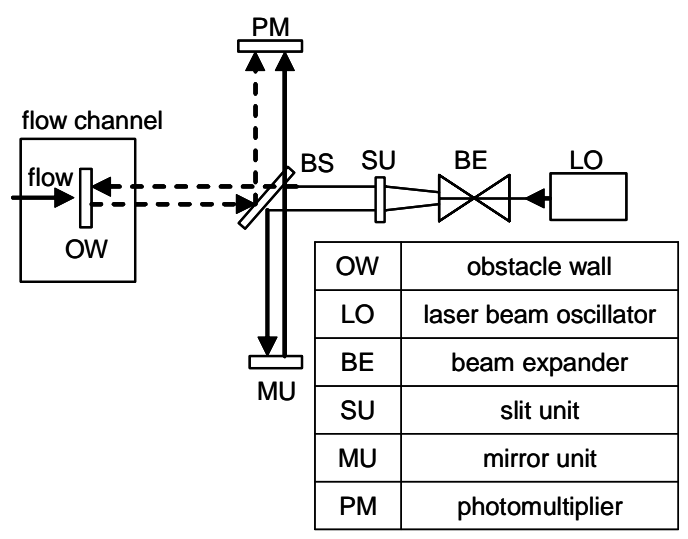

Figure 3. Schematic illustration of the Michelson interferometer used to measure the wall vibration. semitransparent mirror to the photomultiplier. The other first passed through the semitransparent mirror to the mirror on the obstacle wall. The reflected light from the mirror on the obstacle wall continued to the semitransparent mirror and then reflected back into the photomultiplier. Recombination of the two reflected light beams that followed different paths produced an interference fringe. Since spacing of the interference fringes was associated with a difference in the path lengths of two light beams, vibrations in obstacle wall gave rise to alternating patterns of interference fringes. A temporal change in light intensity of interference fringes was detected and amplified by the photomultiplier. A frequency analysis of these data provided the vibration frequency of the obstacle wall.

\subsection{Experimental Conditions}

During the experiments, air was delivered at a steady flow rate of $7.5 \times 10^{-4} \mathrm{~m}^{3} / \mathrm{s}(45 \mathrm{~L} / \mathrm{min})$ to the flow channel by the compressor. The Reynolds number calculated from the constriction diameter and material properties of the air at $15^{\circ} \mathrm{C}$ (density of $1.225 \mathrm{~kg} / \mathrm{m}^{3}$ and viscosity of $\left.1.78 \times 10^{-5} \mathrm{~kg} /(\mathrm{m} \cdot \mathrm{s})\right)$ was 10,269 . Generated aeroacoustic sounds were measured by a microphone that was situated $200 \mathrm{~mm}$ distal to the center of the flow channel. The vibration frequency of the obstacle wall was measured with the Michelson interferometer as described above. Measurements of sound and vibration were synchronized by Labview ver 8.0 (National Instruments). The sampling frequency was 40,000 Hz.

Three experiments were performed under the same flow conditions as described above. In the first experiment, we examined the effects of the presence of an obstacle wall and front cavity on sounds. For this experiment, we prepared three models as summarized in Table 1. In the second experiment, we performed simultaneous measurements of sound pressure and vibration of the obstacle wall to identify vibration-induced sound. In the third experiment, the thickness of the obstacle wall was varied to be $0.29,0.5,1,2$, and $5 \mathrm{~mm}$ to examine the effects of wall rigidity on the sounds.

\subsection{Data Analysis}

A Fourier transformation with a Hanning window was used to attain a frequency spectrum. For smoothening, the amplitude data were averaged in each frequency bin,

Table 1. Models used for the first experiment.

\begin{tabular}{ccccc}
\hline & back cavity & constriction & front cavity & obstacle wall \\
\hline model 1 & + & + & + & + \\
model 2 & + & + & + & - \\
model 3 & + & + & - & -
\end{tabular}

+: Present, -: Absent. 
the center of which was $100+200 \mathrm{~N} \mathrm{~Hz}(\mathrm{~N}=0,1,2 \ldots)$; the bandwidth was $200 \mathrm{~Hz}$. For example, a result at the frequency of $500 \mathrm{~Hz}$ represents an average of the data from 400 to $600 \mathrm{~Hz}$. All results in this paper are presented in this fashion.

Measurements were repeated 5 times in each condition. Although Figure 4 and Figure 5 presented below represent the data of one measurement (not average), reproducibility of the measurements was good enough to give the quantitatively same results.

\section{RESULTS}

\subsection{First Experiment}

Aeroacoustic sounds were measured for three models that differ in assembly as summarized in Table 1. In brief, model 1 was a completely assembled flow channel that had all components, namely, the back cavity, the constriction, the front cavity, and the obstacle wall. The obstacle wall was not present in model 2 , but the rest was the same as model 1. Model 3 lacked both the front cavity and the obstacle wall, and therefore consisted of

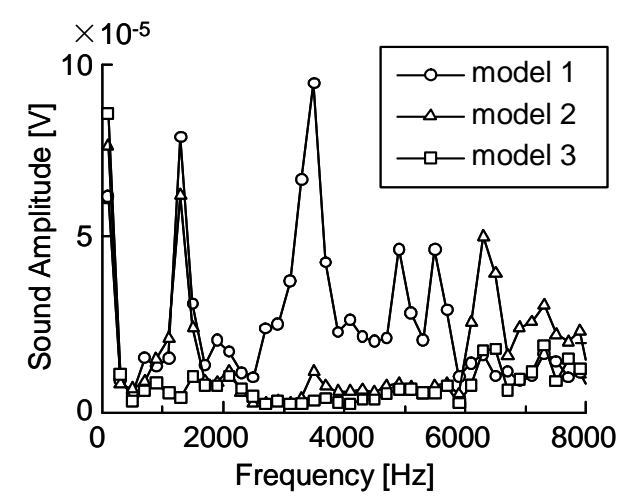

Figure 4. Frequency spectrum of the sound amplitude for model $1(\bigcirc)$, model $2(\Delta)$, and model $3(\square)$. The models differ in their assembly as summarized in Table 1.

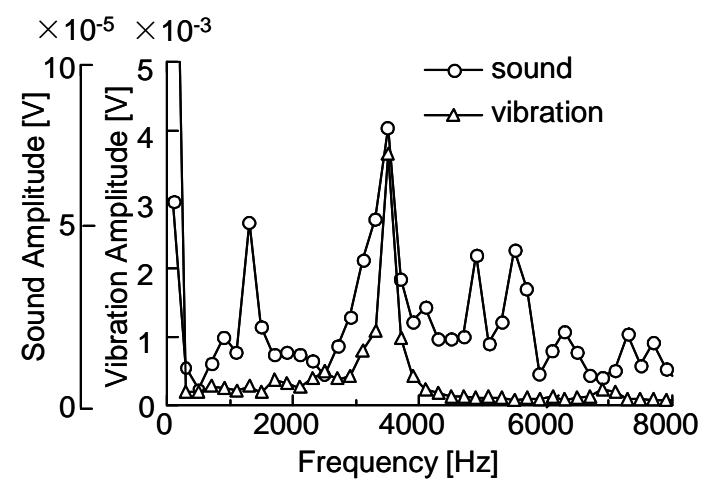

Figure 5. Frequency spectrum of the sound amplitude $(\bigcirc)$ and vibration of the obstacle wall $(\Delta)$. The thickness of obstacle wall was $0.5 \mathrm{~mm}$ and the flow rate was $45 \mathrm{~L} / \mathrm{min}$. the back cavity and the constriction. Figure 4 illustrates the frequency spectrum of the sound pressure amplitudes obtained from the models. The symbols represent model $1(\bigcirc)$, model $2(\Delta)$, and model $3(\square)$. As shown, model 1 , which has all parts including the front cavity, constriction, back cavity, and obstacle wall, exhibited several peaks over the entire frequency range. In particular, prominent peaks were found at the frequencies of 1,300 $\mathrm{Hz}$ and 3,500 Hz. In contrast, model 2, which does not have the obstacle wall, showed a peak at the frequency of $1,300 \mathrm{~Hz}$, but no peak at $3,500 \mathrm{~Hz}$. No peaks were found at these frequencies in model 3 , which only had the front cavity and constriction. A comparison of these results clearly indicates that the sound at $1,300 \mathrm{~Hz}$ is associated with the presence of a front cavity while that at $3,500 \mathrm{~Hz}$ is associated with the presence of an obstacle wall.

\subsection{Second Experiment}

Sound and wall vibrations were simultaneously measured for the model that had all elements. Here, the thickness of the obstacle wall was $0.5 \mathrm{~mm}$ and the flow rate at the inlet was $7.5 \times 10^{-4} \mathrm{~m}^{3} / \mathrm{s}$. Figure 5 plots the frequency spectrum of the sound pressure amplitudes and vibration of the obstacle wall. In this graph, circles and triangles represent sound pressure and vibration of the obstacle wall, respectively. Prominent peaks in sound are observed at the frequencies of $1,300 \mathrm{~Hz}$ and 3,500 $\mathrm{Hz}$, as described in Section 3.1. In contrast, vibration of the obstacle wall showed a sharp peak at the frequency of $3,500 \mathrm{~Hz}$. These results confirmed that sounds at the frequency of $3,500 \mathrm{~Hz}$ are generated by the vibration of the obstacle wall.

\subsection{Third Experiment}

The thickness of the obstacle wall was varied at 0.29, 0.5, 1,2 , and $5 \mathrm{~mm}$ in the model used in Section 3.2. Figure 6 depicts changes in the sound pressure amplitude at the frequencies of (a) $1,300 \mathrm{~Hz}$ and (b) $3,500 \mathrm{~Hz}$ against the thickness of the obstacle wall. Here, the sound amplitude was normalized with the mean amplitude obtained at the thickness of $0.29 \mathrm{~mm}$. The mean and standard deviations are a result of five repeated experiments. We found that the sound at $1,300 \mathrm{~Hz}$ remained almost the same regardless of the thickness of the obstacle wall. Furthermore, the slope of the linear regression analysis was not statistically different from zero in $1,300 \mathrm{~Hz}$. In contrast, the sound at $3,500 \mathrm{~Hz}$ decreased with an increase in wall thickness. In this case, the slope obtained using a linear regression analysis showed a significant difference from zero $(\mathrm{p}<0.05)$, indicating a significant decrease in the amplitude with an increase in the wall thickness. Furthermore, a statistical analysis (t-test) demonstrated a 


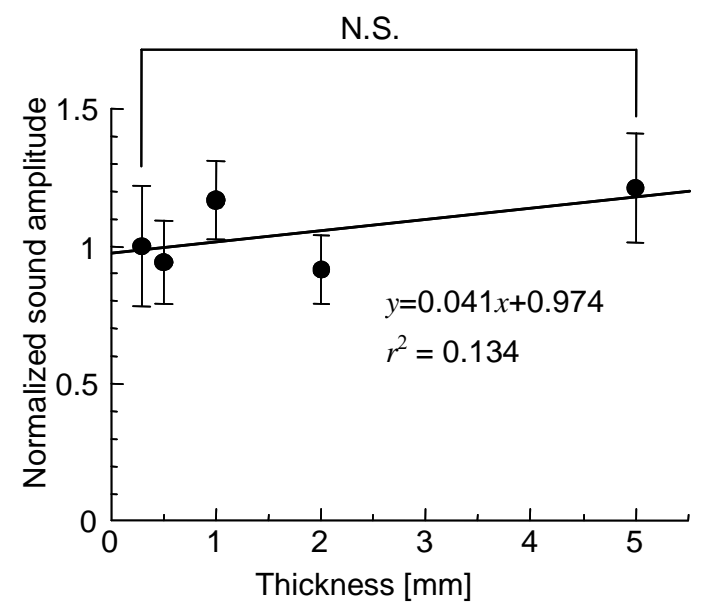

(a) $1,300 \mathrm{~Hz}$

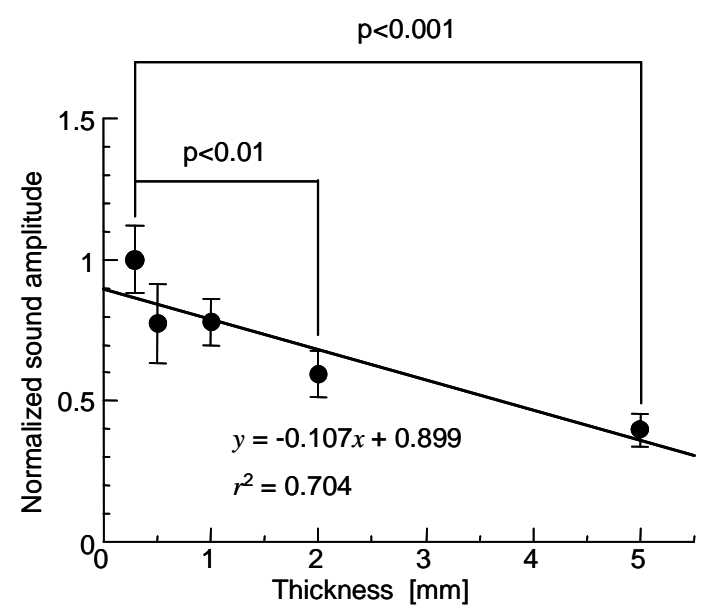

(b) $3,500 \mathrm{~Hz}$

Figure 6. Changes in the sound amplitude at the frequency of (a) $1,300 \mathrm{~Hz}$ and (b) $3,500 \mathrm{~Hz}$ against the thickness of the obstacle wall. All data are normalized with the mean amplitude at a thickness of $0.29 \mathrm{~mm}$. The data are presented as the mean \pm $\mathrm{SD}$ over five randomly implemented experiments.

significant difference in the amplitude between the two thicknesses of $0.29 \mathrm{~mm}$ and $2 \mathrm{~mm}(\mathrm{p}<0.01)$ and between thicknesses of $0.29 \mathrm{~mm}$ and $5 \mathrm{~mm}(\mathrm{p}<0.001)$.

Concomitantly, we measured wall vibrations for all cases. However, wall vibrations were detectable only at the wall thickness of 0.29 and $0.5 \mathrm{~mm}$, probably because the oscillation was too tiny for walls thicker than $0.5 \mathrm{~mm}$. The wall vibration results showed that, for both 0.29 and $0.5 \mathrm{~mm}$, a sharp peak was present at $3,500 \mathrm{~Hz}$ and no other remarkable peaks were observed. The amplitude of vibration was larger for the $0.29 \mathrm{~mm}$ thickness than for $0.5 \mathrm{~mm}$.

\section{DISCUSSION}

Simultaneous measurements of sound pressure and vi- bration provide deep insights into the generation mechanisms of vibration-induced sound. Although it is intuitively obvious that the frequency of vibration is equal to that of sound, it is important to confirm that the obstacle vibrates at the frequency of sound. Here, we used a Michelson interferometer for measuring the vibration of an obstacle. One of the most important features of this method is noninvasiveness to the flow. For measurements, the laser intensity was not too high as to cause thermo-fluid interactions that may alter flows. Another advantage was the capability of measuring the vibration of a target from a distance. Because the laser beam travels in straight lines, it is able to measure the vibration at a distance as long as we do not interrupt or shade the laser beam that strikes and reflects off of the obstacle wall. In addition, theoretically, a spatial resolution of the Michelson interferometer is much smaller than a wavelength of light. Therefore, the Michelson interferometer is suitable for studying vibration-induced sound.

The association between the sound and wall vibration amplitude peaks suggests that the wall vibration would induce sound at 3,500 Hz. We speculate that the airflow injected from the inlet of the flow channel became a jet when passing through the constriction and impinged on the upper edge of the obstacle wall, causing the wall to vibrate and generate the sound. This hypothesis is supported by experiment 3, which demonstrated that the sound amplitude at $3,500 \mathrm{~Hz}$ decreased with increased wall thickness, which actually increased the rigidity of the wall.

To date, it is widely accepted that sibilant sounds /s/ are produced as a result of flow turbulence provoked by teeth positioned in the path of a jet that develops through constriction $[7,9,20]$. In contrast, the tooth vibration has not attained much attention from scientists as a sound source of sibilant /s/, probably because it had been experimentally challenging to assess the wall vibration without disturbing flows. Here, we suggest that tooth vibration can also be a sound source of sibilant $/ \mathrm{s} /$. In reality, an anterior tooth is not as thin as the obstacle wall used in this experiment, which means the teeth are stiff enough to bare fluid dynamic forces induced by the impingement of a jet and turbulence. However, it is speculated that the anterior tooth swings or vibrates in a labio-lingual direction from its root, which is only supported by soft periodontal ligaments. At this moment, beyond demonstrating that sound was generated when the obstacle was present in the path of a jet, we are not able to conclude which source is more dominant for the production of sibilant /s/. Future models of the oral cavity will mimic more realistic oral cavity conditions and examine the effects of the elasticity of obstacles.

The results show that the sound at $1,300 \mathrm{~Hz}$ was not 
the vibration-induced sound. In fact, this sound was generated when the front cavity was present. Since the front cavity of the flow channel has a rigid surface at the inlet (constriction) and is open at the outlet, it can be regarded as a cylinder with one closed end and one open end. In such a cylinder, the acoustic resonance frequency $f$ is calculated by

$$
f=\frac{n v}{4(L+l)},
$$

where $n$ is an odd number, $L$ is the length of the front cavity, $v$ is the speed of sound, and $l$ is the length of the end correction. The end correction is practically given by

$$
l=\alpha r,
$$

where $r$ is the radius of the flow channel $(=12.7 \mathrm{~mm})$ and $\alpha$ is a correction coefficient. According to Levine and Schwinger [21], the correction coefficient $\alpha$ varies from around 0.15 to 0.6133 depending on the ratio of the wavelength to the radius of the flow channel. Given the length of the front cavity $(=60 \mathrm{~mm})$, the length of the end correction $l$ is $1.905-7.789 \mathrm{~mm}$. Consequently, assuming that the speed of sound $v$ is $343 \mathrm{~m} / \mathrm{s}$, we predict that acoustic resonance occurs at the frequency of 1,264$1,385 \mathrm{~Hz}$ or its integral multiple in this flow channel. This acoustic resonance frequency falls in the frequency band of 1,200-1,400 Hz represented by its central frequency of $1,300 \mathrm{~Hz}$. Therefore, we speculate that the sound at $1,300 \mathrm{~Hz}$ was a result of the acoustic resonance in the front cavity.

The present experiment has some limitations. First, the thickness of the mirror $(0.5 \mathrm{~mm})$ attached to the obstacle wall to ensure the reflection of the light beam for vibration measurements is comparable to that of obstacle wall. This may have resulted in losing intrinsic nature of the wall vibration. To overcome this problem, we may need to use a negligibly thin mirror or polish the surface of the obstacle wall like a mirror. Second, the present setup of the Michelson interferometer is not capable of measuring the displacement amplitude in length metrics like a "micrometer" and analyzing spatial variations over the wall vibration. This information would be essential to gain a deeper insight into the generation mechanisms of vibration-induced sound. Third, due to this averaging procedure, we might have lost information regarding the frequency shift of sound pressure and vibration of the obstacle wall with changing wall thicknesses. Although we have examined various bandwidths of the frequency to the averaged data to determine if such a shift in the frequency with changes in the wall thickness occurred, it was not observed. Future studies will employ more sophisticated means to minimize measurement errors such that we can explore the relationship between wall vibra- tion and sound pressure in detail. Notwithstanding these limitations, the results in this paper suggest the potential of the presented methodology to pave the way for detailed analysis of vibration-induced sound.

\section{SUMMARY AND CONCLUSION}

In the present study, we performed simultaneous measurements of aeroacoustic sounds and obstacle wall vibration utilizing an optical technique called the Michelson interferometer. The flow channel, which simplified the geometry of the mouth cavity, generated aeroacoustic sounds, given airflows from the inlet. The results demonstrate two prominent peaks in sound at 1,300 and $3,500 \mathrm{~Hz}$, and one peak in the wall vibration at $3,500 \mathrm{~Hz}$. The association between peak frequencies of sound and wall vibration suggested that the sound at $3,500 \mathrm{~Hz}$ is induced by wall vibration. In fact, the amplitude of sound at 3,500 Hz decreased with thickening of the obstacle wall, which also increased its rigidity. The experiments demonstrate that the developed techniques are capable of measuring aeroacoustic sound and obstacle wall vibration simultaneously. Future studies will adopt a more complex geometry of the oral cavity and explore the effects of obstacle wall rigidity on generated sounds, while ameliorating fixation of the obstacle to the model and measurements of wall vibration with the Michelson interferometer. These results will provide us with additional insights into the production of vibration-induced sounds and sibilant sounds $/ \mathrm{s} /$.

\section{ACKNOWLEDGEMENTS}

This work was supported in part by the Global COE Program "In Silico Medicine-oriented Worldwide Open Platform" at Osaka University.

\section{REFERENCES}

[1] Lee, S.Y.A., Whitebill, L.T., Ciocca, V. and Samman, N. (2002) Acoustic and perceptual analysis of the sibilant sound /s/ before and after orthognathic surgery. Journal of Oral and Maxillofacial Surgery, 60, 364-373.

[2] Hamlet, S. Geoffrey, V.D. and Bartlett, D.M. (1976) Effect of a dental prosthesis on speaker-specific characteristics of voice. Journal of Speech and Hearing Research, 19, 639-650.

[3] Banky, J. (2000) Mouthguards dental injury and problems: On-field management. Journal of Science and Medicine in Sport, 3, 5-11. doi:10.1016/S1440-2440(00)80063-8

[4] Fletcher, S.G. and Newman, D.G. (1991) [s] and [sh] as a function of linguapalatal contact place and sibilant. Journal of the Acoustical Society of America, 89, 850858. doi:10.1121/1.1894646

[5] Čelebić, A. and Knezović-Zlatarić, D. (2003) A comparison of patient's satisfaction between complete and partial removable denture wearers. Journal of Dentistry, 31, 
445-451. doi:10.1016/S0300-5712(03)00094-0

[6] Hassan, T., Naini, F.B. and Gill, D. S. (2007) The effects of orthognathic surgery on speech: A review. Journal of Oral and Maxillofacial Surgery, 65, 2536-2543. doi:10.1016/j.joms.2007.05.018

[7] Shadle, C.H. (1990) Articulatory-acoustic relationships in fricative consonants. In: Hardcastle W.J. and Marchal A. Eds., Speech Production and Speech Modeling, Kluwer Academic Publishers, The Netherlands, 187-209.

[8] Ray, D.K. and Charles R.D., (2002) The Acoustic Analysis of Speech, 2nd Edition, Singular/Thomson Learning, Canada, 38-43.

[9] Stevens, K.N. (1971) Airflow and turbulence noise for fricative and stop consonants: Static considerations. Journal of the Acoustical Society of America, 50, 1180-1192. doi:10.1121/1.1912751

[10] Stevens, K.N. (1988) Acoustic Phonetics, MIT Press, Cambridge, MA, 379-412.

[11] Shadle, C.H. (1985) The acoustics of fricative consonants, MIT Technical Report, Cambridge, MA.

[12] Shadle, C.H. (1991) The effect of geometry on source mechanisms of fricative consonants. Journal of Phonetics, 19, 409-424.

[13] Howe, M. and McGowan, R. (2005) Aeroacoustics of [s], Proceedings of Royal Society, London Series A, 461, 1005-1028. doi:10.1098/rspa.2004.1405

[14] Nozaki K., Tamagawa, H. and Shimojo, S. (2008) Prediction of dental fricative sound by Lighthill-Curle equation. In: Takada, K. Ed., In Silico Dentistry, Medigit, Japan, 155-158.

[15] Lighthill, M.J. (1952) On sound generation aerodynamically I. General theory. Proceedings of Royal Society, London Series A, 211, 564-587. doi:10.1098/rspa.1952.0060

[16] Curle, N. (1955) The influence of solid boundaries upon aerodynamic sound. Proceedings of Royal Society, London Series A, 231, 505-514. doi:10.1098/rspa.1955.0191

[17] Van Hirtum, A., Grandchamp, X., Pelorson, X., Nozaki, K. and Shimojo, S. (2010) LES simulation and 'in-vitro' experimental validation of flow around a teeth-shaped obstacle. International Journal of Applied Mechanics, 2, 265-279. doi:10.1142/S1758825110000603

[18] Ju, L., Blair, D.G. and Zhau C. (2000) Detection of gravitational waves. Reports on Progress in Physics, 63, 1317-1427. doi:10.1088/0034-4885/63/9/201

[19] Narayanan, S., Alwan, A. and Haker K. (1995) An articulartory study of fricative consonants using magnetic resonance imaging. Journal of the Acoustical Society of America, 98, 1325-1347. doi:10.1121/1.413469

[20] Fant, G. (1960) Acoustic theory of speech production, The Hague, The Netherlands, Mouton.

[21] Levine, H. and Schwinger, J. (1948) On the radiation of sound from an unflanged circular pipe. Physical Review, 73, 383-406. doi:10.1103/PhysRev.73.383 